\title{
Histone deacetylase 1 facilitates aerobic glycolysis and growth of endometrial cancer
}

\author{
QIONGWEI WU, WENYING ZHANG, YU LIU, YUHUA HUANG, HUIHENG WU and CHENGBIN MA \\ Gynecology Department, Shanghai Changning Maternity and Infant Health Hospital, Shanghai 200051, P.R. China
}

Received March 1, 2021; Accepted June 24, 2021

DOI: 10.3892/ol.2021.12982

\begin{abstract}
The deregulation of histone deacetylase 1 (HDAC1) is reportedly involved in the progression of several cancer types. However, its function in endometrial cancer remains unknown. The aim of the present study was to clarify the role of HDAC1 in aerobic glycolysis and the progression of endometrial cancer. Lentiviral vector transfection was used to up- and downregulate HDAC1 expression in HEC-1-A endometrial cancer cells. The effects of HDAC1 on cellular proliferation, apoptosis, migration, invasiveness and tumorigenesis were determined by CCK-8, flow cytometry, wound-healing, transwell chamber and in vivo tumor formation experiments, respectively. HDAC1 level was significantly increased in endometrial cancer tissues and cells, and its high expression was associated with advanced clinicopathological progression. HEC-1-A cell proliferation, invasiveness, migration and tumorigenesis were enhanced, and apoptosis was inhibited when HDAC1 was overexpressed. Moreover, upregulation of HDAC1 significantly promoted the epithelial-mesenchymal transition of HEC-1-A cells, and increased glucose consumption, lactate secretion and ATP levels. Collectively, the present study revealed that HDAC1 promoted the aerobic glycolysis and progression of endometrial cancer, which may provide a potential target for endometrial cancer treatment.
\end{abstract}

\section{Introduction}

Endometrial cancer is the most common gynecologic malignancy in the United States, with $>63,000$ new cases in 2018 (1). Benefiting from early detection and advanced therapies, patients with endometrial cancer have shown improved survival rates during the past few decades (2). However, the cure rate is significantly low, and the prognosis is poor for patients with advanced metastatic disease (3). Therefore,

Correspondence to: Dr Chengbin Ma, Gynecology Department, Shanghai Changning Maternity and Infant Health Hospital, 786 Yuyuan Road, Changning, Shanghai 200051, P.R. China E-mail:macb66@126.com

Key words: histone deacetylase 1, growth, epithelial-mesenchymal transition, aerobic glycolysis, migration further investigation into the mechanisms underlying the metastasis of endometrial cancer, with the aim to develop effective treatment methods and improve patient prognosis, is crucial.

Aerobic glycolysis, the Warburg effect, is a primary characteristic of cancer cells $(4,5)$. Tumor cells exhibit increased biosynthesis through glucose uptake and lactate production via the aerobic glycolysis pathway, thus are characterized by rapid proliferation, invasiveness and metastatic potential (6). Emerging evidence has shown that the deregulation of aerobic glycolysis plays an important role in cellular proliferation, invasiveness and angiogenesis of cancers, including cervical cancer $(7,8)$. Therefore, repression of aerobic glycolysis may be a potential therapeutic method for preventing cancer progression (9).

Numerous genes and factors have been strongly implicated in the progression of endometrial cancer, such as p53 and p16 inactivation, PTEN mutations, and the upregulation of estrogen and/or progesterone receptors (10-12). Histone deacetylases (HDACs) are important enzymes which induce deacetylation, resulting in an inactive chromatin structure. Notably, the genetic alterations in endometrial cancer are strongly affected by histone-mediated epigenetics, including histone deacetylation, indicating that HDACs may play an important role in the progression of endometrial cancer (10-12). For example, Li et al (13) found that the administration of romidepsin (FK228), a HDAC inhibitor, impaired cellular proliferation and accelerated apoptosis by increasing p53 expression in endometrial cancer Ishikawa and HEC-1-A cells. De et al (14) reported that MHY2256, another HDAC inhibitor, induced apoptosis and autophagy in endometrial cancer cells by elevating p53 acetylation. Furthermore, Zheng et al (15) found that HDAC6 was overexpressed in endometrial cancer, and that this promoted cellular proliferation, invasiveness and metastatic potential. HDAC1, together with HDAC2, 3 and 8, are members of the Class I of HDACs family (16). Evidence has demonstrated that HDAC1 exerts an oncogenic role in various human tumor types, including breast $(17,18)$, lung (19) and ovarian cancer (20). Tang et al (18) demonstrated that HDAC1 was upregulated in breast cancer cells, where it promoted proliferation and migration via upregulation of interleukin-8. Liu et al (20) reported that HDAC1-knockdown suppressed cellular proliferation, and increased apoptosis and chemosensitivity in cisplatin-resistant A2780/CDDP ovarian cancer cells. Moreover, Cao et al (19) found that HDAC1 
mRNA and protein expression was closely associated with the differentiation grade of lung cancer. Also, the expression level of HDAC1 in gastrointestinal malignancies, especially in colorectal cancer, was found to be higher than that in noncancerous tissues, which was closely associated with advanced tumor stage and poor prognosis (21). In endometrial cancer, Weichert et al (22) suggested that the majority of endometrial cancer cells are characterized by elevated expression of HDAC1, and that the expression levels of HDAC1 are associated with cellular proliferative capacity. However, the role of HDAC1 in the occurrence and progression of endometrial cancer remains unknown.

The aim of the present study was to reveal the effects of HDAC1 on the proliferation, migration, aerobic glycolysis and tumorigenesis of endometrial cancer by modulating the level of HDAC1 via knockdown or overexpression.

\section{Materials and methods}

Patients. The present study was approved by the ethical committee of Shanghai Changning Maternity and Infant Health Hospital (approval. no. CNFBLLKT-2017-011), and adheres to the Declaration of Helsinki. In total, 64 paired endometrial cancer and adjacent-normal tissues were obtained from patients diagnosed with endometrial cancer at the Shanghai Changning Maternity and Infant Health Hospital (Shanghai, China) between February 2010 and February 2013. The patient age range was from 34 to 76 years. Clinicopathological characteristics, including age, parity, body mass index, International Federation of Gynecology and Obstetrics [FIGO] stage (23), differentiation and metastasis status, as well as the survival time after surgery, were all obtained from electronic medical records. Inclusion criteria: Patients underwent surgery prior to chemoradiotherapy, and provided written informed consent. Exclusion criteria: i) Patients complicated with epithelial ovarian cancer or other malignant tumors; ii) Patients had received chemoradiotherapy prior to surgery; and iii) Patients who didn't provide written informed consent. The relative mRNA level of HDAC $1 \geq$ the mean was considered as high $\mathrm{HDAC} 1$ expression, and $<$ the average value was considered as low HDAC1 expression.

Cell culture. Normal human endometrium cells from non-malignant myoma (KC02-44D hTERT), endometrial cancer cell lines HEC-1-A and HEC-1-B, and 293T cells were all obtained from the American Type Culture Collection (ATCC). All cells were maintained in Dulbecco's modified Eagle's medium, supplemented with $10 \%$ fetal bovine serum (FBS) and 1\% penicillin/streptomycin (all Thermo Fisher Scientific, Inc.) at $37^{\circ} \mathrm{C}$ with $5 \% \mathrm{CO}_{2}$.

Cell transfection and treatment. The lentivirus vectors used to upregulate or downregulate HDAC1 in human endometrial cancer HEC-1-A cells are termed overexpression (OE)-HDAC1 (cat. no. RC201745L4V) and short hairpin (sh)-HDAC1 (cat. no. TL312496V), and were purchased from OriGene Technologies, Inc. 293T cells were used to generate the virions through transfection with vectors $(20 \mu \mathrm{g})$, phelper $1.0(15 \mu \mathrm{g})$ and phelper $2.0(10 \mu \mathrm{g})$ (BioVector NTCC, Inc.) using Lipofectamine ${ }^{\circledR} 2000$ reagent (Invitrogen; Thermo Fisher
Scientific, Inc.) at $37^{\circ} \mathrm{C}$. Viral particles were collected $72 \mathrm{~h}$ post-transfection, then centrifuged at $1,776 \mathrm{xg}$ for $5 \mathrm{~min}$ at $4^{\circ} \mathrm{C}$, and filtered with $0.45 \mu \mathrm{m}$ filter. For cell infection, HEC-1-A cell culture medium was added with OE-HDAC1, sh-HDAC1, or their negative control vectors (OE-NC, sh-NC) with a multiplicity of infections of 5, 6, 5 and 6 for $6 \mathrm{~h}$, followed by replacement with fresh medium and incubation at $37^{\circ} \mathrm{C}$ for another $24 \mathrm{~h}$. To construct stably-transfected cell lines, $5 \mu \mathrm{g} / \mathrm{ml}$ puromycin was added to the culture medium after $24 \mathrm{~h}$ of transfection and incubated for 14 days at $37^{\circ} \mathrm{C}$. The shRNA sequences are as follows: Sh-HDAC1-1 Sense, 5'-CACCGAGAAAGACCCAGAGGA GAAGCTCGAGCTTCTCCTCTGGGTCTTTCTC-3' and antisense, 5'-AAAAGAGAAAGACCCAGAGGAGAAGCT CGAGCTTCTCCTCTGGGTCTTTCTC-3'; Sh-HDAC1-2 sense, 5'-CACCGAAGAAAGAAGTCACCGAAGACTCGA GTCTTCGGTGACTTCTTTCTTC-3' and antisense, 5'-AAA AGAAGAAAGAAGTCACCGAAGACTCGAGTCTTCGGT GACTTCTTTCTTC-3'.

Reverse transcription-quantitative (RT-q) PCR. Total RNA was extracted from tissues and cells using the RNApure Tissue \& Cell Kit (DNase I) according to the manufacturer's instructions (CWBio). The RNA samples were then subjected to cDNA synthesis and qPCR using the SuperRT One Step RT-PCR Kit (CWBio) on a Bio-Rad detection system (Bio-Rad Laboratories, Inc.). $\beta$-actin expression was used to normalize the mRNA levels of HDAC1, which were calculated using the $2^{-\triangle \Delta C q}$ method (24). The qPCR thermocycling conditions were as follows: Initial denaturation at $95^{\circ} \mathrm{C}$ for $30 \mathrm{sec}$, followed by annealing and elongation for 40 cycles of $95^{\circ} \mathrm{C}$ for $15 \mathrm{sec}, 60^{\circ} \mathrm{C}$ for $30 \mathrm{sec}$ and $72^{\circ} \mathrm{C}$ for $30 \mathrm{sec}$, and a final extension at $72^{\circ} \mathrm{C}$ for 2 min. Primers targeting HDAC1 and $\beta$-actin were obtained from Invitrogen (Thermo Fisher Scientific, Inc.), the sequences of which are as follows: HDAC1 forward, 5'-TGCTAAAGT ATCACCAGAGGGT-3' and reverse, 5'-TGGCCTCATAGG ACTCGTCA-3'; and $\beta$-actin forward, 5'-ACAGAGCCTCGC CTTTGCC-3' and reverse, 5'- CACACTTGGCGTGTCCT TTG-3'.

Western blotting. Total protein samples extracted from tissues and cells were obtained using RIPA lysis buffer containing $50 \mathrm{mM}$ Tris (pH 7.4), $150 \mathrm{mM} \mathrm{NaCl}, 1 \%$ Triton X-100, $1 \%$ sodium deoxycholate, $0.1 \%$ sodium dodecyl sulfonate, sodium orthovanadate, sodium fluoride, EDTA and leupeptin. The proteins were quantified using a BCA Protein Assay kit (Thermo Fisher Scientific, Inc.). Subsequently, 20-30 $\mu \mathrm{g}$ protein from each sample was separated by SDS-PAGE with $10 \%$ polyacrylamide gels, and then transferred onto PVDF membranes (MilliporeSigma). The membranes were blocked with $5 \%$ non-fat milk for $1 \mathrm{~h}$ at room temperature, and probed with anti-HDAC1 (1:2,000; cat. no. \#5356; Cell Signaling Technology, Inc.), anti-E-cadherin (1:1,000; cat. no. ab15148; Abcam), anti-N-cadherin (1:1,000; cat. no. ab18203; Abcam), anti-cleaved caspase-3 (1:1,000; cat. no. ab2302; Abcam), anti-hypoxia-inducible factor 1 (HIF-1) $\alpha(1: 3,000$; cat. no. ab1; Abcam), anti-pyruvate kinase PKM (1:5,000; cat. no. ab85555; Abcam), anti-L-lactate dehydrogenase A chain (1:5,000; cat. no. ab101562; Abcam) and anti- $\beta$-actin $(1: 5,000$; cat. no. ab8226; Abcam) antibodies overnight at $4^{\circ} \mathrm{C}$. Then, the membranes were incubated with the corresponding secondary 
antibodies, mouse anti-rabbit IgG-HRP (cat. no. sc-2357) and goat anti-mouse IgG-HRP (cat. no. sc-2005) (both Santa Cruz Biotechnology, Inc.) for $1 \mathrm{~h}$ at room temperature. Protein signaling was enhanced using ECL reagents (MilliporeSigma) and then examined using a western blotting imaging and quantitation system (Bio-Rad Laboratories, Inc.). ImageJ software (v1.8.0; National Institutes of Health) was used to quantify protein expression.

Cell Counting Kit-8 (CCK-8) assay. To assess cellular proliferation capacity, HEC-1-A cells were first seeded into 96 -well plates at a density of $3 \times 10^{3}$ cells/well, and cultured at $37^{\circ} \mathrm{C}$ overnight, followed by lentiviral infection with sh-HDAC1, sh-NC, OE-HDAC1, OE-NC. For proliferative assessment, $10 \mu \mathrm{l}$ CCK-8 reagent (Beyotime Institute of Biotechnology) and $90 \mu \mathrm{l}$ fresh medium was added to each well, and the cells were incubated for another $4 \mathrm{~h}$ at $37^{\circ} \mathrm{C}$. The absorbance was recorded at $450 \mathrm{~nm}$ every $24 \mathrm{~h}$ using a plate reader (Bio-Rad Laboratories, Inc.).

Flow cytometry. The effects of HDAC1 on apoptosis were assessed by flow cytometry using the Annexin V (FITC)/Propidium Iodide (PI) apoptosis detection kit (Nanjing KeyGen Biotech Co., Ltd.) according to manufacturer's instructions. Then, apoptosis rates were detected with a CytoFLEX flow cytometer (Beckman Coulter, Inc.) and analyzed using FlowJo 7.6 software (FlowJo LLC).

Wound-healing assay. HEC-1-A cells (1x105/well) infected with sh-HDAC1, sh-NC, OE-HDAC1 or OE-NC were seeded into 24 -well plate and incubated at $37^{\circ} \mathrm{C}$ until confluent. Then, wounds were created across each monolayer with a $20-\mu 1$ pipette tip, and the floating cells were removed. Subsequently, the cells were placed at $37^{\circ} \mathrm{C}$ and cultured for another $24 \mathrm{~h}$ with serum-free medium (Thermo Fisher Scientific, Inc.). The wound width was recorded using an inverted microscope (magnification, $\mathrm{x} 40$ ) at 0 and $24 \mathrm{~h}$ after wound formation.

Transwell assay. Transwell chambers (8.0- $\mu \mathrm{m}$; Corning, Inc.) were precoated with $50 \mu \mathrm{l}$ Matrigel (at a ratio of 1:1 with culture medium) for $1 \mathrm{~h}$ at $37^{\circ} \mathrm{C}$, and then used to assess

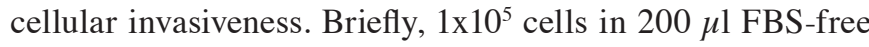
culture medium (Thermo Fisher Scientific, Inc.) were placed into the upper chamber of each well, and $600 \mu \mathrm{l}$ medium supplemented with $15 \%$ FBS was added into the lower chamber. After incubation for $24 \mathrm{~h}$ at $37^{\circ} \mathrm{C}$, cells at the upper surface of the membrane were removed with cotton swabs, while cells on the lower surface were fixed with $4 \%$ paraformaldehyde for $15 \mathrm{~min}$ at room temperature, followed by staining with $0.1 \%$ crystal violet solution (Beyotime Institute of Biotechnology) for $10 \mathrm{~min}$ at room temperature. Then, the invasive cells were counted under an inverted microscope with a magnification of $\mathrm{x} 200$.

Detection of lactate production, glucose consumption and ATP levels. Lactate production, glucose consumption and ATP levels were measured according to a previous report (25). A total of $2 \times 10^{5}$ cells were seeded into each well of 6 -well plates and maintained at $37^{\circ} \mathrm{C}$ for $48 \mathrm{~h}$, after which cellular lactate production was measured using a Lactate Colorimetric Assay
Kit (cat. no. K627; BioVision, Inc.). The cells were incubated in cell culture medium without FBS for $1 \mathrm{~h}$ at $37^{\circ} \mathrm{C}$, and the supernatant was collected for measurement of lactate production. The reaction mixture was incubated for $30 \mathrm{~min}$ at room temperature and protected from light, and the lactate levels were measured at $450 \mathrm{~nm}$ using a microplate reader.

Glucose consumption was assessed using the Glucose Uptake Colorimetric Assay Kit (cat. no. K676; BioVision, Inc.). Following lentivirus infection, cells were cultured at $37^{\circ}$ for $48 \mathrm{~h}$, collected and seeded into 96 -well plates with $1 \times 10^{4}$ cells per well. Then, the cells were glucose-starved by preincubating with Krebs-Ringer-Phosphate-HEPES buffer $(100 \mu \mathrm{l})$ for $40 \mathrm{~min}$, and incubated with $10 \mu \mathrm{l}$ 2-deoxyglucose $(10 \mathrm{mM})$ for $20 \mathrm{~min}$, followed by Reaction Mix A for $1 \mathrm{~h}$ at $37^{\circ} \mathrm{C}$. Then, $90 \mu \mathrm{l}$ extraction buffer was added to each well, and incubated at $90^{\circ} \mathrm{C}$ for $40 \mathrm{~min}$, and then placed in an ice bath for $5 \mathrm{~min}$. Then, Reaction Mix B was added to each well, followed by centrifugation at $16,000 \mathrm{x}$ g at $4^{\circ} \mathrm{C}$ for $2 \mathrm{~min}$. The $\mathrm{OD}$ value of the supernatant at $412 \mathrm{~nm}$ was measured using a microplate analyzer.

An ATP Colorimetric Assay Kit (cat. no. MAK1900; Sigma-Aldrich; Merck KGaA) was used for ATP level measurement according to the manufacturer's protocol. Cells $\left(5 \times 10^{5}\right)$ were collected and added to $100 \mu$ l ATP Assay Buffer. The cells were centrifuged at $16,000 \mathrm{x} \mathrm{g}$ for $5 \mathrm{~min}$ at room temperature and the supernatant was used for ATP measurement. The reaction mixture was incubated for $30 \mathrm{~min}$ at room temperature, protected from light, and measured at $570 \mathrm{~nm}$ in a microplate reader.

Animal experiments. Animal experiments were performed in accordance with the National Institute of Health Guidelines for the Care and Use of Laboratory Animals, and were approved by the Animal Care and Research Committee of Shanghai Changning Maternity and Infant Health Hospital. Female, 6-week-old BALB/c athymic nude mice (Beijing Vital River Laboratory Animal Technology) were housed in a specific pathogen-free animal facility with free access to water and food, at $22 \pm 1^{\circ} \mathrm{C}$ with $55 \pm 2 \%$ humidity under a $12 \mathrm{~h}$ light/dark cycle. To construct the tumor-bearing mouse model, $1 \times 10^{6}$ sh-HDAC1, OE-HDAC1, sh-NC or OE-NC stably transfected HEC-1-A cells were subcutaneously injected into the nude mice. A total of 12 mice were used, and three mice were included in each group. The animal health, behavior and tumor growth were monitored every 3 days; the tumors were weighed four weeks post cancer cell injection. The mice were euthanized by cervical dislocation following 28 days of injection unless the volume reached $1,000 \mathrm{~mm}^{3}$, at which point the mice were sacrificed early. Mice were considered as dead when no heartbeat and breathing were observed, and reflexes were absent. Tumor volume was calculated according to the following formula: Volume = length $\mathrm{x}$ width $2 / 2$.

Statistical analysis. With the exception of the animal study, a total of three independent experiments were performed per assay, and the data are expressed as mean \pm standard deviation. SPSS 22.0 software (IBM Corp.) was used to performed data analysis. The association between HDAC1 expression and the clinicopathological feature of patients with endometrial cancer was assessed using the $\chi^{2}$ test. Data conformed to 

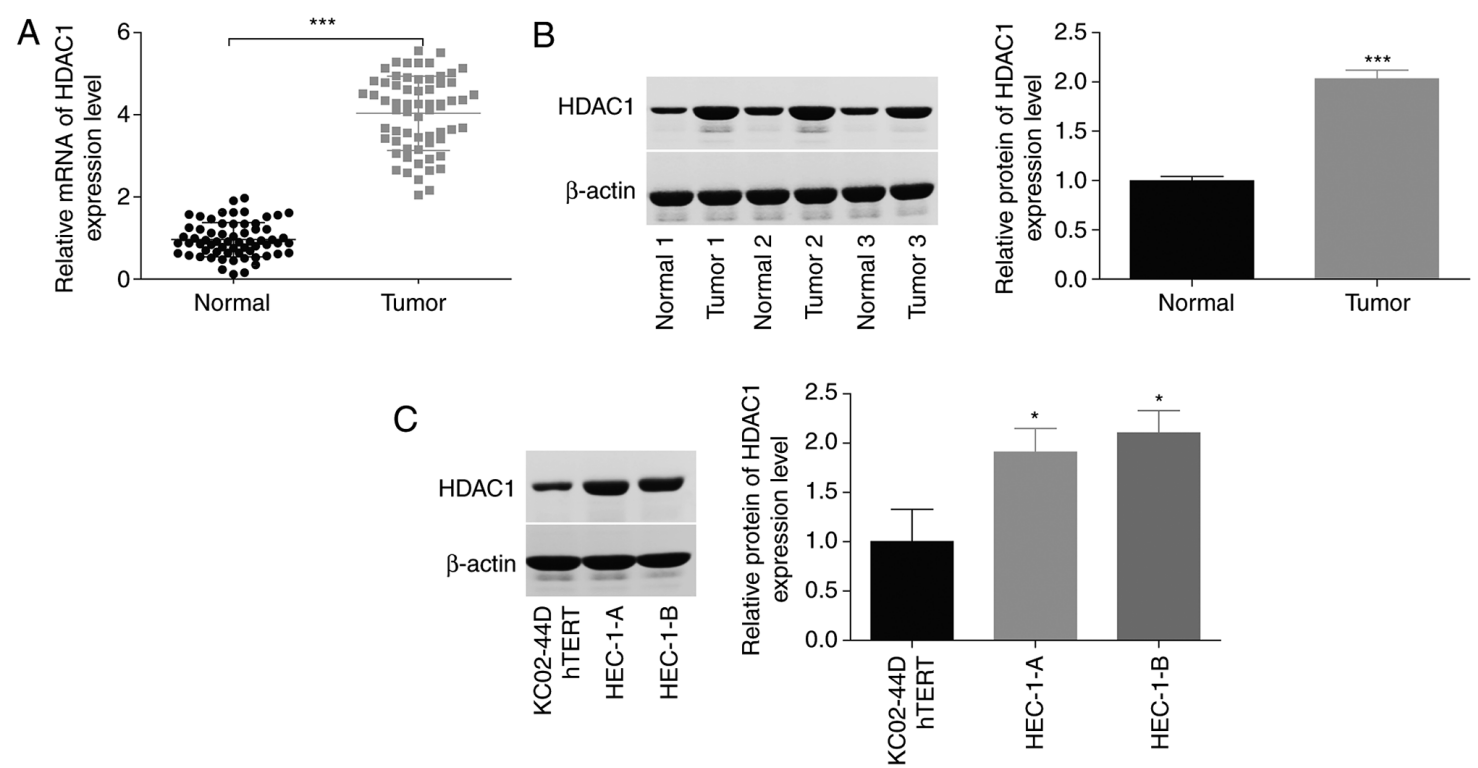

Figure 1. Assessment of HDAC1 expression levels in endometrial cancer and matched-normal tissues. (A) Reverse transcription-quantitative PCR and (B) western blot analysis revealed that HDAC1 expression at both the mRNA (64 paired tumors) and protein (3 paired tissues) level was significantly increased in endometrial cancer tissues, compared with normal tissues. Normal 1/2/3 and Tumor 1/2/3 are representative of the normal tissue and cancer tissue samples from 64 cases. ${ }^{* * *} \mathrm{P}<0.001$, paired Student's t-test. (C) Protein levels of HDAC1 in HEC-1-A, HEC-1-B and KC02-44D hTERT cells were determined by western blotting. $\mathrm{N}=3$; ${ }^{*} \mathrm{P}<0.05$, ANOVA followed by Tukey test. HDAC1, histone deacetylase 1 .

Gaussian distribution (determined by Shapiro-Wilk test), and the t-test and one-way ANOVA followed by Tukey's test were used for data analysis between 2 groups or multiple groups, respectively, after homogeneity testing of variance using the F test. Paired Student's t test was applied for data comparisons between cancer and matched-normal tissues. $\mathrm{P}<0.05$ was considered to indicate a statistically significant difference.

\section{Results}

HDACl expression is significantly increased in endometrial cancer tissues and cells. To reveal the function of HDAC1 in the progression of endometrial cancer, the expression patterns of HDAC1 were assessed in endometrial cancer tissues and cells. As shown by RT-qPCR and western blotting, a significant increase ( 3 -fold) in HDAC1 expression level was observed in cancer tissues compared with normal tissue samples (Fig. 1A and B). In addition, HDAC1 expression levels in human endometrial cancer cell lines (HEC-1-A and HEC-1-B) were 2-fold that of those in KC02-44D hTERT cells (Fig. 1C). These results indicated that HDAC1 was highly expressed in endometrial cancer.

HDAC1 promotes cellular proliferation and inhibits apoptosis in endometrial cancer. The function of HDAC1 in HEC-1-A cell proliferation and apoptosis was then investigated. The expression levels of HDAC1 were reduced by $50-70 \%$ at the mRNA and protein levels following infection with sh-HDAC1-1 and sh-HDAC1-2 (Fig. 2A and B). By contrast, HDAC1 expression was increased by $100-250 \%$ in HEC-1-A cells infected with OE-HDAC1 compared with OE-NC (Fig. 2C and D). Upregulation of HDAC1 induced a 1.5-fold increase in cellular proliferation (Fig. 2E) and inhibited apoptosis from $5.8 \pm 0.6 \%$ to $2.6 \pm 0.5 \%$ (Fig. $2 \mathrm{~F}-\mathrm{G}$ ), whereas downregulation of HDAC1 impaired cellular proliferation and induced cell apoptosis (Fig. 2E-G) compared with the corresponding control group. In addition, downregulation of HDAC1 induced an $80 \%$ increase in cleaved caspase-3 expression, while upregulation decreased HDAC1 expression (Fig. 2H). These findings suggested that HDAC1 facilitated cellular proliferation and inhibited apoptosis in endometrial cancer.

HDACl triggers cellular migration and invasiveness in endometrial cancer. In addition, the effects of HDAC1 on the modulation of cellular migration and invasiveness in endometrial cancer. Compared with the control group, HEC-1-A cell migration and invasiveness were enhanced by 55 and $80 \%$ following infection with OE-HDAC1, whereas the migration and invasiveness of HEC-1-A cells were inhibited by 50 and $55 \%$ when infected with sh-HDAC1 (Fig. 3A and B). Due to the crucial role of epithelial-mesenchymal transition (EMT) in cancer cell migration $(26,27)$, the potential effects of HDAC1 on EMT regulation in endometrial cancer were investigated. The expression levels of $\mathrm{N}$-cadherin, a mesenchymal cell marker, were elevated by $\sim 100 \%$, while the expression of E-cadherin, an epithelial cell marker, was decreased by $55 \%$ when HDAC1 was overexpressed; sh-HDAC1-1/-2 transfection resulted in the opposite effect (Fig. 3C). These results suggest that HDAC1 serves as a promoter of cellular migration and invasiveness in endometrial cancer.

HDACl induces aerobic glycolysis in endometrial cancer cells. HDAC1 function in the aerobic glycolysis of endometrial cancer cells was subsequently investigated. The glucose consumption, lactate secretion and ATP level were all increased by $\sim 1$-fold following HEC-1-A cell infection with 
A

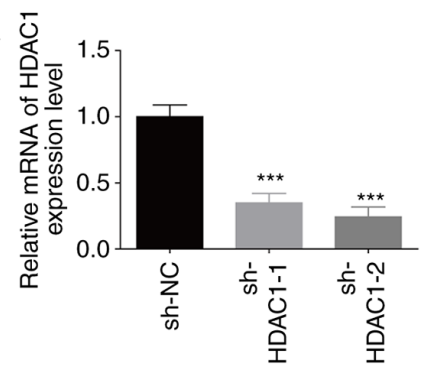

B

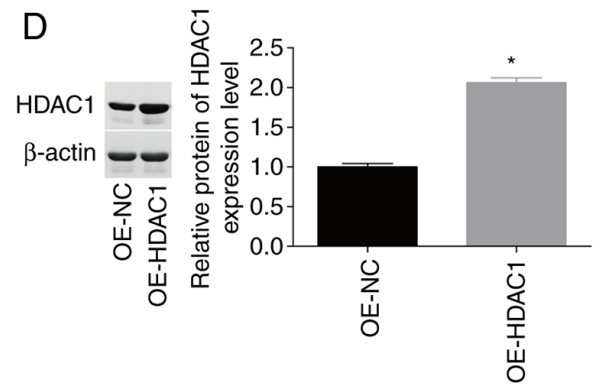

G

$\overline{\mathbf{x}}$

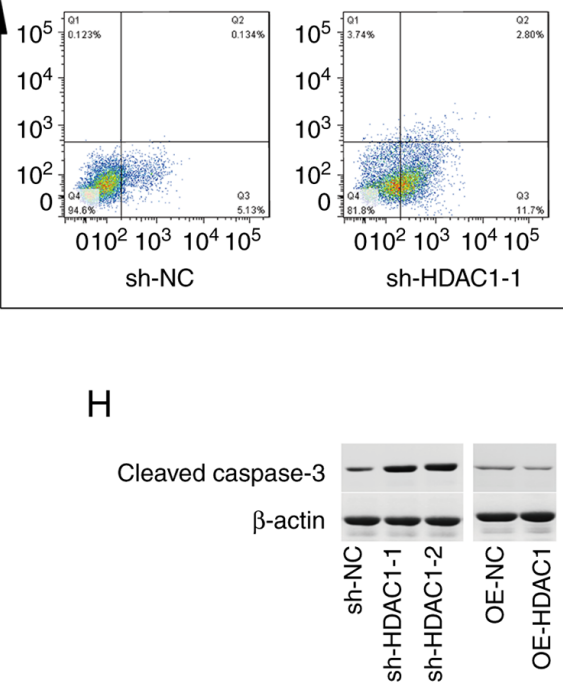

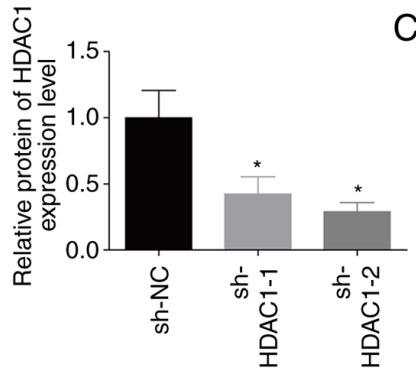

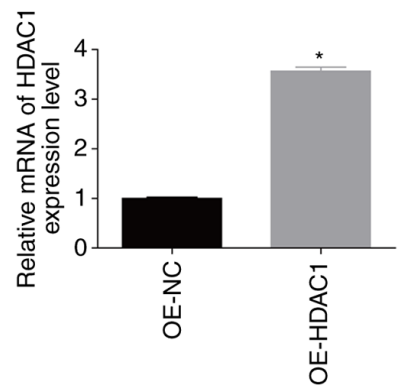

E

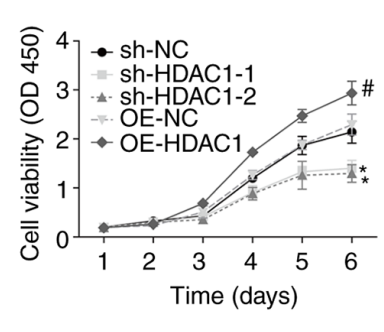

F

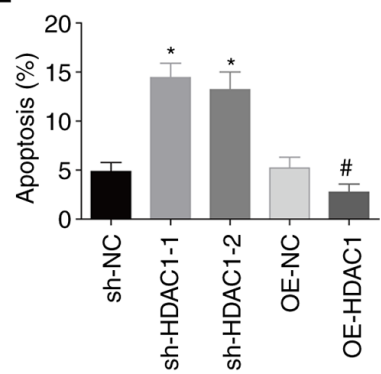

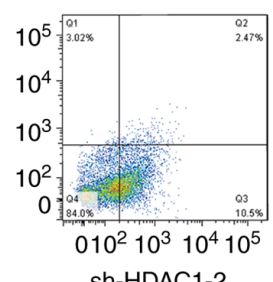
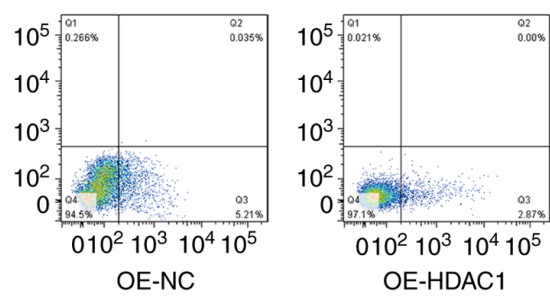

FITC

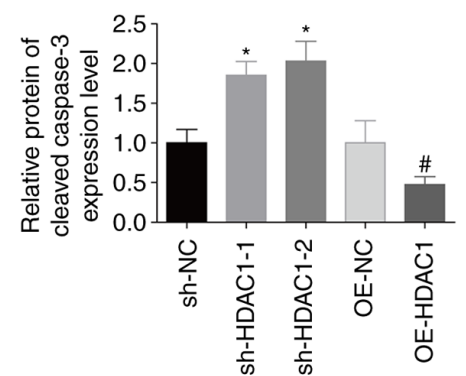

Figure 2. Evaluation of HDAC1 effects on cellular proliferation and apoptosis. HEC-1-A cells were infected with sh-HDAC1-1/-2, sh-NC, OE-HDAC1 or OE-NC, prior to experimentation. (A-D) Reverse transcription-quantitative PCR and western blotting were performed to detect HDAC1 mRNA and protein levels. (E) Cell Counting Kit 8 analysis showed that HDAC1 downregulation inhibited cellular proliferation, which was promoted by OE-HDAC1. (F and G). Annexin V FITC/PI staining showed that sh-HDAC1 transfection induced apoptosis and OE-HDAC1 repressed apoptosis. (H) Expression levels of cleaved caspase- 3 were determined by western blotting. $\mathrm{N}=3$; ANOVA followed by Tukey's test; ${ }^{*} \mathrm{P}<0.05,{ }^{* * * *} \mathrm{P}<0.001$ sh-HDAC 1 vs. sh-NC; ${ }^{*} \mathrm{P}<0.05$, OE-HDAC1 vs. OE-NC. HDAC1, histone deacetylase 1; sh, short hairpin; OE, overexpression; PI, propidium iodide.

OE-HDAC1, and decreased by 40-60\% when HDAC1 was downregulated (Fig. 4A-C). In addition, HDAC1 overexpression induced significant increases in HIF-1 $\alpha$, PKM2 and LDHA levels, while downregulation of HDAC1 decreased the expression levels of these proteins, compared with the control group (Fig. 4D). These findings suggested that HDAC1 induced aerobic glycolysis in endometrial cancer.

HDACl promotes endometrial cancer growth in vivo. In addition, the role of HDAC1 in endometrial cancer growth in vivo was investigated. Compared with the control group, tumor volume and weight 28 days post-cell injection were increased by 90 and $120 \%$ when HDAC1 was overexpressed in HEC-1-A cells, and decreased by 40 and $60 \%$ when
HDAC1 was downregulated, respectively (Fig. 5A-C). This assay confirmed that HDAC1 promoted endometrial cancer growth in vivo.

High expression of HDACl is closely associated with the advanced clinicopathological features of patients with endometrial cancer. The clinical value of HDAC1 in endometrial cancer was then evaluated. As shown in Table I, it was observed that high HDAC1 expression level was significantly associated with poor differentiation $(\mathrm{P}=0.005)$, advanced FIGO stage $(\mathrm{P}=0.019)$, higher incidence rate of lymphatic metastasis $(\mathrm{P}=0.009)$ and deeper muscular wall invasion depth $(\mathrm{P}=0.043)$, indicating the potential of HDAC1 as a diagnosed marker for endometrial cancer. 
A

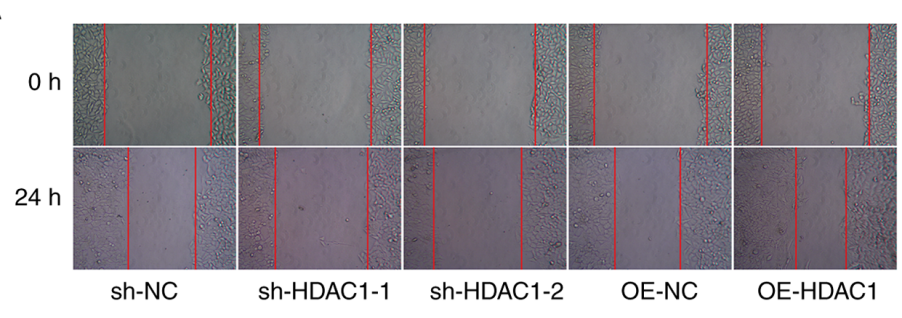

B

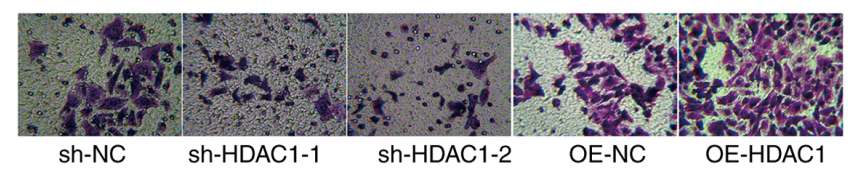

C

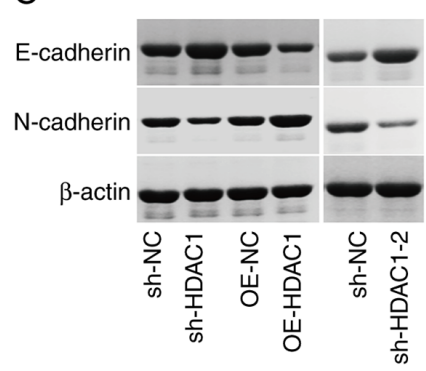

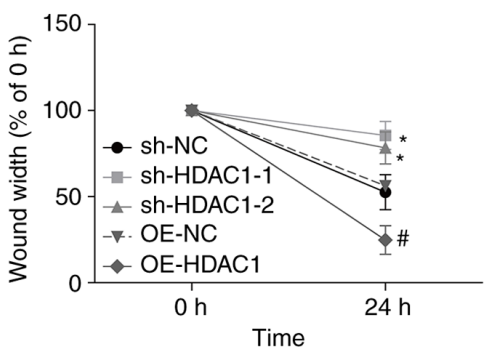
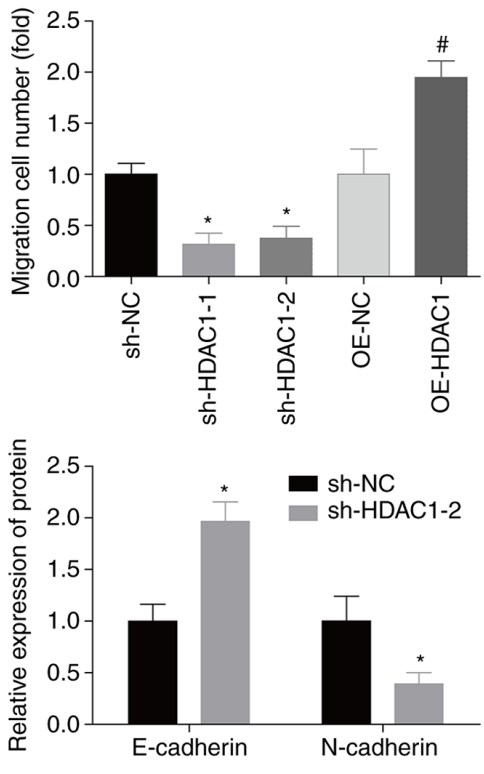

Figure 3. Evaluation of HDAC1 effects on the migration and invasiveness of endometrial cancer cells. (A) Wound-healing assays were used to assess the effects of sh-HDAC1-1/-2 and OE-HDAC1 on HEC-1-A cell migration (Magnification: 40). (B) Transwell assays were performed to evaluate the effects of sh-HDAC1-1/-2 and OE-HDAC1 on HEC-1-A cell invasiveness (Magnification: 200). (C) Western blotting was used to detect the protein expression levels of $\mathrm{N}$-cadherin and E-cadherin. $\mathrm{N}=3$; ANOVA followed by Tukey's test; $\mathrm{P}<0.05$, sh-HDAC1 vs. sh-NC; ${ }^{\mathrm{P}} \mathrm{P}<0.05$, OE-HDAC1 vs. OE-NC). HDAC1, histone deacetylase 1; sh, short hairpin; OE, overexpression.
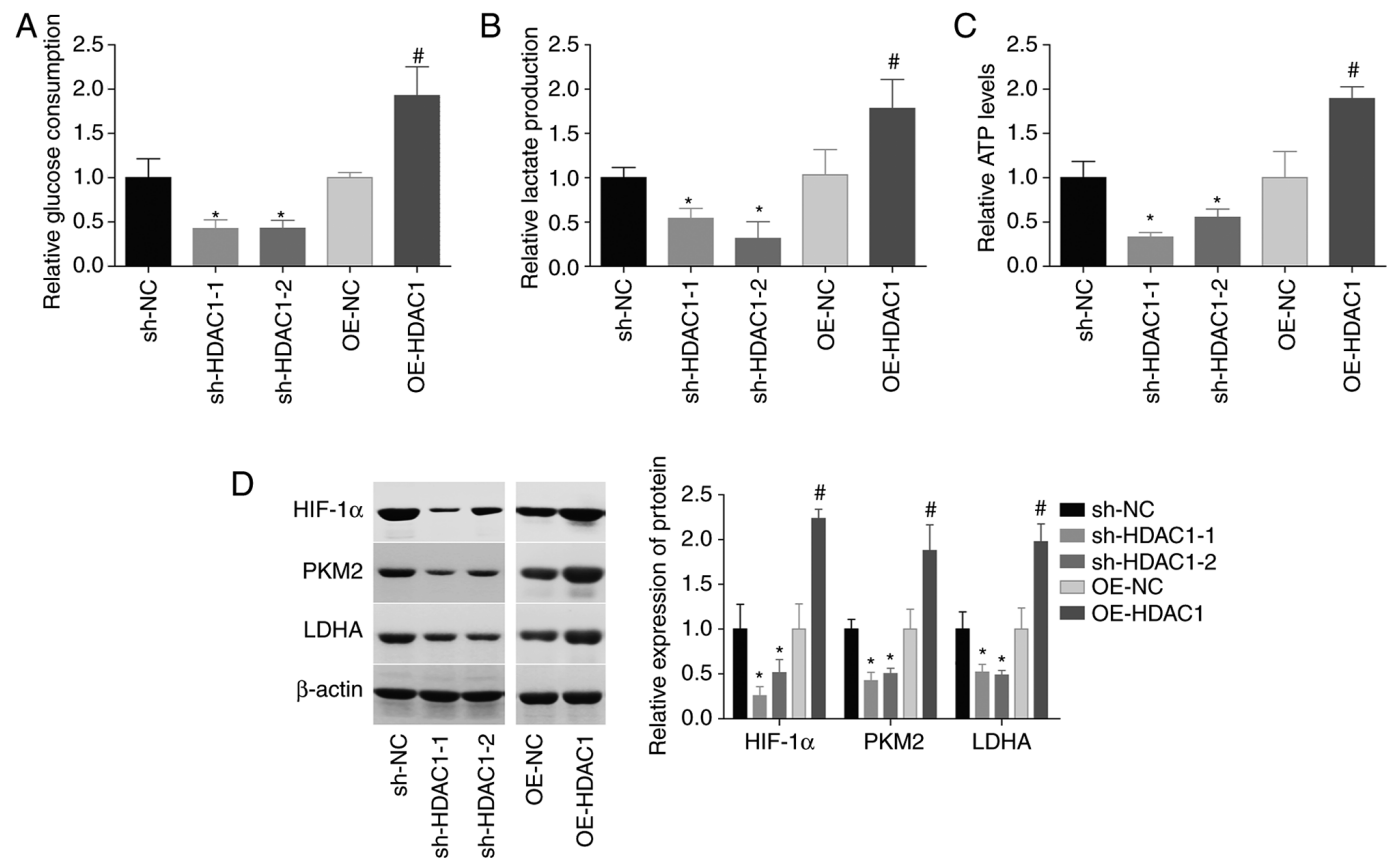

Figure 4. HDAC1 induces aerobic glycolysis in endometrial cancer cells. (A) Glucose consumption, (B) lactate secretion and (C) ATP levels were assessed in HEC-1-A cells infected with OE-NC, OE-HDAC1, sh-NC or sh-HDAC1-1/-2. (D) Expression levels of HIF-1 $\alpha$, PKM2 and LDHA were measured by western blotting. $\mathrm{N}=3$, ANOVA followed by Tukey test; ${ }^{\prime} \mathrm{P}<0.05$, sh-HDAC1 group vs. sh-NC group; ${ }^{\prime} \mathrm{P}<0.05$, OE-HDAC1 group vs. OE-NC group). HDAC1, histone deacetylase 1; sh, short hairpin; OE, overexpression; HIF-1 $\alpha$, hypoxia-inducible factor 1; PKM2, pyruvate kinase PKM; LDHA, L-lactate dehydrogenase A chain. 
Table I. Evaluation of the clinical value of histone deacetylase 1 in endometrial cancer.

\begin{tabular}{|c|c|c|c|}
\hline Index & High expression $(n=37)$ & Low expression $(n=27)$ & P-value \\
\hline Age, years & $35-75$ & $34-76$ & 0.449 \\
\hline$\leq 60$ & 22 & 13 & \\
\hline$>60$ & 15 & 14 & \\
\hline Degree of differentiation & & & 0.005 \\
\hline Well & 14 & 20 & \\
\hline Moderate-poor & 23 & 7 & \\
\hline FIGO stage & & & 0.019 \\
\hline I-II & 17 & 21 & \\
\hline III-IV & 20 & 6 & \\
\hline Lymphatic metastasis & & & 0.009 \\
\hline Negative & 18 & 22 & \\
\hline Positive & 19 & 5 & \\
\hline Muscular wall invasion depth & & & 0.043 \\
\hline$<1 / 2$ & 16 & 19 & \\
\hline$\geq 1 / 2$ & 21 & 8 & \\
\hline
\end{tabular}

FIGO, international federation of gynecology and obstetrics.
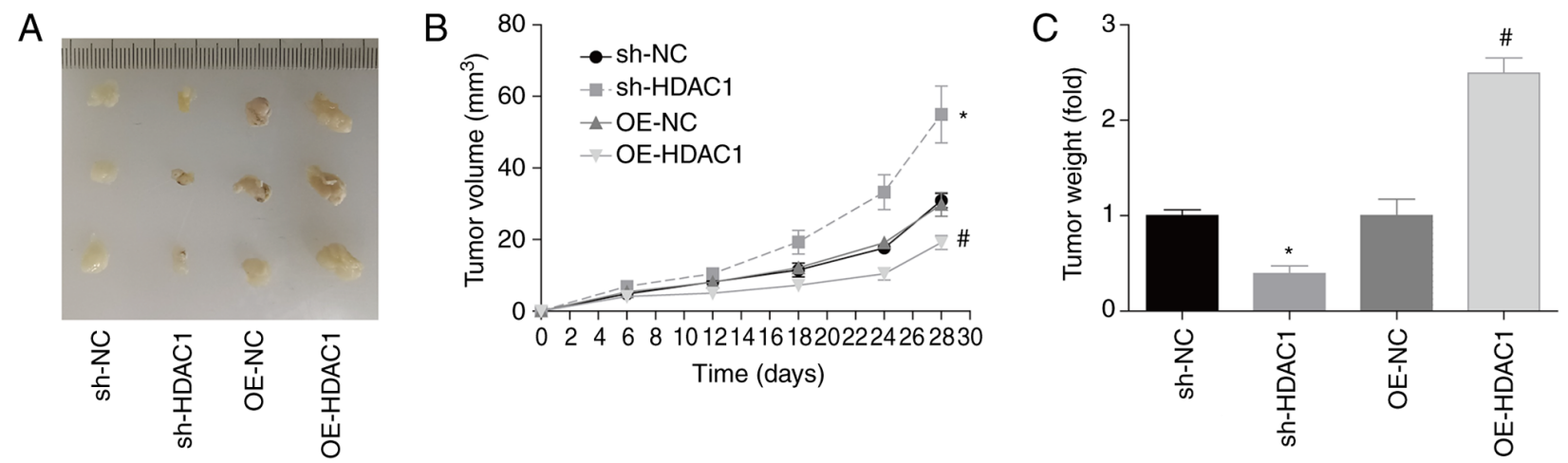

Figure 5. HDAC1 induces tumorigenesis in endometrial cancer. Mice were injected with HEC-1-A cells which were stably transfected with OE-NC, OE-HDAC1, sh-NC or sh-HDAC1-1. (A) Tumor images. (B) Tumor volume and (C) weight were assessed. A total of three mice were included in each group; ANOVA followed by Tukey's test; "P<0.05, sh-HDAC1 vs. sh-NC; "P<0.05, OE-HDAC1 vs. OE-NC. HDAC1, histone deacetylase 1; sh, short hairpin; OE, overexpression.

\section{Discussion}

Through the regulation of a wider spectrum of substrate proteins, HDACs have been identified to be closely involved in a range of cellular processes, such as viability, survival, apoptosis and differentiation $(28,29)$, and have been identified as potential targets for the treatment of multiple cancer types, (30,31), including endometrial cancer (32). In the present study, the role HDAC1 in endometrial cancer progression was investigated. Consistent with the work of Weichert et al (22), HDAC1 expression was found to be significantly elevated in endometrial cancer tissues and cells, compared with adjacent-normal tissues and control cells. In addition, the high expression level of HDAC1 was closely linked to a malignant clinical phenotype in patients with endometrial cancer. Similarly, HDAC1 expression was closely associated with the differentiation grade of lung cancer (19). Also, the expression level of HDAC1 in gastrointestinal malignancies, especially in colorectal cancer, was higher than that in noncancerous tissues, which closely was associated with advanced tumor stage and poor prognosis (21).

To date, the role of HDAC1 has been revealed in various types of cancer (33). For instance, HDAC1 was upregulated in breast cancer, and HDAC1 overexpression induced cellular proliferation and migration, while HDAC1 silencing inhibited proliferation $(17,18)$. Liu et al $(20)$ reported that HDAC1-knockdown suppressed cellular proliferation, and increased apoptosis and chemosensitivity in cisplatin-resistant ovarian cancer A2780/CDDP cells. Downregulation of HDAC1 also induced apoptosis in advanced thyroid cancer cells (34), as well as inhibiting invasiveness and inducing apoptosis in non-small cell lung cancer cells (35). These aforementioned 
studies have demonstrated that HDAC1 serves as an oncogene in multiple cancer types. Similarly, in the current study, the role of HDAC1 in modulating cellular function was investigated in an endometrial cancer setting. Consistent with other cancer types, the results showed that the upregulation of HDAC1 significantly promoted proliferation, tumorigenesis, migration and invasiveness abilities, and repressed apoptosis in HEC-1-A cells, indicating that HDAC1 exerts an oncogenic role in endometrial cancer.

EMT is a key process in cancer metastasis $(26,27)$, and studies have shown that HDAC inhibitors play different roles in EMT in various cancers. HDAC inhibitors induced EMT in colon carcinoma $(36,37)$, but have also been shown to revert EMT in non-small cell lung (38), biliary tract (39), prostate (40) and head and neck cancers (41). These findings suggest that the regulatory role of HDACs during EMT is associated with the cancer type. In the present study, the role of HDAC1 in endometrial cancer cell EMT was investigated. The results demonstrated that the expression of $\mathrm{N}$-cadherin was significantly elevated, while that of E-cadherin was decreased when HEC-1-A cells were infected with OE-HDAC1; sh-HDAC1 caused an opposing effect, indicating that HDAC1 accelerated EMT, which then contributed to cancer cell migration.

To further elucidate the mechanisms underlying the effects of HDAC1 in endometrial cancer, its effect on glycolysis [through which cancer cells obtain additional energy to maintain rapid growth and migration $(8,42)]$ were assessed. The results showed that HDAC1 overexpression significantly increased the glucose consumption, lactate secretion and ATP level of HEC-1-A cells, suggesting that HDAC1 induces aerobic glycolysis in endometrial cancer. Aerobic glycolysis may be the mechanism by which HDAC1 accelerates endometrial cancer progression. Chen et al (43) reported that HDAC1 increased the expression of HIF-1 $\alpha$ in colorectal cancer. HIF-1 $\alpha$ is a well-known master regulator of glycolysis and can enhance the expression of glycolytic genes (25). Consistently, the effects of HDAC1 on HIF-1 $\alpha$ expression were assessed in the present study, and revealed that HDAC1 overexpression promoted HIF-1 $\alpha$ expression, indicating that HDAC1 potentially facilitated aerobic glycolysis in endometrial cancer through HIF-1 $\alpha$. To this end, rescue experiments should be carried out in the future.

In conclusion, to the best of our knowledge, the present study was the first to reveal that HDAC1 facilitates aerobic glycolysis and growth in endometrial cancer, which may provide a potential target for endometrial cancer treatment.

\section{Acknowledgements}

Not applicable.

\section{Funding}

The present study was supported by the Fund Project of the Changning District Science and Technology Commission (grant. no. CNKW2017Y16).

\section{Availability of data and materials}

All data generated or analyzed during this study are included in this published article.

\section{Authors' contributions}

CM conceived the project and revised the manuscript. QW, WZ and YL conducted the experiments. QW wrote the paper. QW, YH and HW analyzed the data. CM and QW confirmed the authenticity of all the raw data. All authors have read and approved the final manuscript.

\section{Ethics approval and consent to participate}

The present study was approved by the ethical committee of Shanghai Changning Maternity and Infant Health Hospital (approval. no. CNFBLLKT-2017-011). Written consent was obtained from all patients.

\section{Patient consent for publication}

Not applicable.

\section{Competing interests}

The authors declare that they have no competing interests.

\section{References}

1. Siegel RL, Miller KD and Jemal A: Cancer statistics, 2018. CA Cancer J Clin 68: 7-30, 2018.

2. Morice P, Leary A, Creutzberg C, Abu-Rustum N and Darai E: Endometrial cancer. Lancet 387: 1094-1108, 2016.

3. Ma J, Li D, Kong FF, Yang D, Yang $\mathrm{H}$ and Ma XX: miR-302a-5p/367-3p-HMGA2 axis regulates malignant processes during endometrial cancer development. J Exp Clin Cancer Res 37: 19, 2018.

4. Chen G, Zhang Y, Liang J, Li W, Zhu Y, Zhang M, Wang C and Hou J: Deregulation of hexokinase II is associated with glycolysis, autophagy, and the epithelial-mesenchymal transition in tongue squamous cell carcinoma under hypoxia. Biomed Res Int 2018: 8480762, 2018.

5. Chen G, Liu H, Zhang Y, Liang J, Zhu Y, Zhang M, Yu D, Wang $C$ and Hou J: Silencing PFKP inhibits starvation-induced autophagy, glycolysis, and epithelial mesenchymal transition in oral squamous cell carcinoma. Exp Cell Res 370: 46-57, 2018.

6. Lee M and Yoon JH: Metabolic interplay between glycolysis and mitochondrial oxidation: The reverse Warburg effect and its therapeutic implication. World J Biol Chem 6: 148-161, 2015.

7. Han J, Zhang L, Guo H, Wysham WZ, Roque DR, Willson AK, Sheng X, Zhou C and Bae-Jump VL: Glucose promotes cell proliferation, glucose uptake and invasion in endometrial cancer cells via AMPK/mTOR/S6 and MAPK signaling. Gynecol Oncol 138: 668-675, 2015.

8. Han X, Ren C, Yang T, Qiao P, Wang L, Jiang A, Meng Y, Liu Z, Du Y and Yu Z: Negative regulation of AMPKalphal by PIM2 promotes aerobic glycolysis and tumorigenesis in endometrial cancer. Oncogene 38: 6537-6549, 2019.

9. Ganapathy-Kanniappan S: Molecular intricacies of aerobic glycolysis in cancer: Current insights into the classic metabolic phenotype. Crit Rev Biochemi Mol Biol 53: 667-682, 2018.

10. Mackay HJ, Eisenhauer EA, Kamel-Reid S, Tsao M, Clarke B, Karakasis K, Werner HM, Trovik J, Akslen LA, Salvesen HB, et al: Molecular determinants of outcome with mammalian target of rapamycin inhibition in endometrial cancer. Cancer 120: 603-610, 2014.

11. Gehrig PA, Le LV, Olatidoye B and Geradts J: Estrogen receptor status, determined by immunohistochemistry, as a predictor of the recurrence of stage I endometrial carcinoma. Cancer 86: 2083-2089, 1999.

12. Myers AP: New strategies in endometrial cancer: Targeting the $\mathrm{PI} 3 \mathrm{~K} / \mathrm{mTOR}$ pathway-the devil is in the details. Clin Cancer Res 19: 5264-5274, 2013. 
13. Li LH, Zhang PR, Cai PY and Li ZC: Histone deacetylase inhibitor, romidepsin (FK228) inhibits endometrial cancer cell growth through augmentation of p53-p21 pathway. Biomed Pharmacother 82: 161-166, 2016.

14. De U, Son JY, Sachan R, Park YJ, Kang D, Yoon K, Lee BM, Kim IS, Moon HR and Kim HS: A new synthetic histone deacetylase inhibitor, MHY2256, induces apoptosis and autophagy cell death in endometrial cancer cells via p53 acetylation. Int J Mol Sci 19: 2743, 2018

15. Zheng Y, Yang X, Wang C, Zhang S, Wang Z, Li M, Wang Y, Wang $X$ and Yang $X$ : HDAC6, modulated by miR-206, promotes endometrial cancer progression through the PTEN/AKT/mTOR pathway. Sci Rep 10: 3576, 2020.

16. Weichert W: HDAC expression and clinical prognosis in human malignancies. Cancer Lett 280: 168-176, 2009.

17. Senese S, Zaragoza K, Minardi S, Muradore I, Ronzoni S, Passafaro A, Bernard L, Draetta GF, Alcalay M, Seiser C and Chiocca S: Role for histone deacetylase 1 in human tumor cell proliferation. Mol Cell Biol 27: 4784-4795, 2007.

18. Tang Z, Ding S, Huang H, Luo P, Qing B, Zhang S and Tang R: HDAC1 triggers the proliferation and migration of breast cancer cells via upregulation of interleukin-8. Biol Chem 398 1347-1356, 2017

19. Cao LL, Song X, Pei L, Liu L, Wang H and Jia M: Histone deacetylase HDAC1 expression correlates with the progression and prognosis of lung cancer: A meta-analysis. Medicine (Baltimore) 96: e7663, 2017.

20. Liu X, Yu Y, Zhang J, Lu C, Wang L, Liu P and Song H: HDAC1 silencing in ovarian cancer enhances the chemotherapy response. Cell Physiol Biochem 48: 1505-1518, 2018.

21. Cao LL, Yue Z, Liu L, Pei L, Yin Y, Qin L, Zhao J, Liu H, Wang H and Jia M: The expression of histone deacetylase HDAC1 correlates with the progression and prognosis of gastrointestinal malignancy. Oncotarget 8: 39241-39253, 2017.

22. Weichert W, Roske A, Niesporek S, Noske A, Buckendahl AC Dietel M, Gekeler V, Boehm M, Beckers T and Denkert C: Class I histone deacetylase expression has independent prognostic impact in human colorectal cancer: Specific role of class histone deacetylases in vitro and in vivo. Clin Cancer Res 14: 1669-1677, 2008

23. Prat J: FIGO staging for uterine sarcomas. Int J Gynaecol Obstet 104: 177-178, 2009.

24. Livak KJ and Schmittgen TD: Analysis of relative gene expression data using real-time quantitative PCR and the 2(-Delta Delta C(T)) method. Methods 25: 402-408, 2001

25. Li L, Liang Y, Kang L, Liu Y, Gao S, Chen S, Li Y, You W, Dong Q, Hong T, et al: Transcriptional regulation of the Warburg effect in cancer by SIX1. Cancer Cell 33: 368-385, 2018.

26. Liu F, Gu LN, Shan BE, Geng CZ and Sang MX: Biomarkers for EMT and MET in breast cancer: An update. Oncol Lett 12 4869-4876, 2016.

27. Nantajit D, Lin D and Li JJ: The network of epithelial-mesenchymal transition: Potential new targets for tumor resistance. J Cancer Res Clin Oncol 141: 1697-1713, 2015.

28. Yang XJ and Seto E: HATs and HDACs: From structure, function and regulation to novel strategies for therapy and prevention. Oncogene 26: 5310-5318, 2007.

29. Villagra A, Sotomayor EM and Seto E: Histone deacetylases and the immunological network: Implications in cancer and inflammation. Oncogene 29: 157-173, 2010.
30. Glozak MA and Seto E: Histone deacetylases and cancer. Oncogene 26: 5420-5432, 2007.

31. Stazi G, Fioravanti R, Mai A, Mattevi A and Valente S: Histone deacetylases as an epigenetic pillar for the development of hybrid inhibitors in cancer. Curr Opin Chem Biol 50: 89-100, 2019.

32. Garmpis N, Damaskos C, Garmpi A, Spartalis E, Kalampokas E, Kalampokas T, Margonis GA, Schizas D, Andreatos N, Angelou A, et al: Targeting histone deacetylases in endometrial cancer: A paradigm-shifting therapeutic strategy? Eur Rev Med Pharmacol Sci 22: 950-960, 2018.

33. Khabele D, Son DS, Parl AK, Goldberg GL, Augenlicht LH, Mariadason JM and Rice VM: Drug-induced inactivation or gene silencing of class I histone deacetylases suppresses ovarian cancer cell growth: Implications for therapy. Cancer Biol Ther 6: 795-801, 2007.

34. Lin CL, Tsai ML, Lin CY, Hsu KW, Hsieh WS, Chi WM, Huang LC and Lee CH: HDAC1 and HDAC2 double knockout triggers cell apoptosis in advanced thyroid cancer. Int J Mol Sci 20: 454, 2019.

35. Zhang L, Bu L, Hu J, Xu Z, Ruan L, Fang Y and Wang P: HDAC1 knockdown inhibits invasion and induces apoptosis in non-small cell lung cancer cells. Biol Chem 399: 603-610, 2018.

36. Ji M, Lee EJ, Kim KB, Kim Y, Sung R, Lee SJ, Kim DS and Park SM: HDAC inhibitors induce epithelial-mesenchymal transition in colon carcinoma cells. Oncol Rep 33: 2299-2308, 2015.

37. Feng J, Cen J, Li J, Zhao R, Zhu C, Wang Z, Xie J and Tang W: Histone deacetylase inhibitor valproic acid (VPA) promotes the epithelial mesenchymal transition of colorectal cancer cells via up regulation of snail. Cell Adh Migr 9: 495-501, 2015.

38. Jakobsen KR, Demuth C, Sorensen BS and Nielsen AL: The role of epithelial to mesenchymal transition in resistance to epidermal growth factor receptor tyrosine kinase inhibitors in non-small cell lung cancer. Transl Lung Cancer Res 5: 172-182, 2016.

39. Sakamoto T, Kobayashi S, Yamada D, Nagano H, Tomokuni A, Tomimaru Y, Noda T, Gotoh K, Asaoka T, Wada H, et al: A histone deacetylase inhibitor suppresses epithelial-mesenchymal transition and attenuates chemoresistance in biliary tract cancer. PLoS One 11: e0145985, 2016.

40. Ruscetti M, Dadashian EL, Guo W, Quach B, Mulholland DJ, Park JW, Tran LM, Kobayashi N, Bianchi-Frias D, Xing Y, et al: HDAC inhibition impedes epithelial-mesenchymal plasticity and suppresses metastatic, castration-resistant prostate cancer. Oncogene 35: 3781-3795, 2016.

41. Bruzzese F, Leone A, Rocco M, Carbone C, Piro G, Caraglia M, Gennaro ED and Budillon A: HDAC inhibitor vorinostat enhances the antitumor effect of gefitinib in squamous cell carcinoma of head and neck by modulating ErbB receptor expression and reverting EMT. J Cell Physiol 226: 2378-2390, 2011.

42. Lunt SY and Heiden MG: Aerobic glycolysis: Meeting the metabolic requirements of cell proliferation. Ann Rev Cell Dev Biol 27: 441-464, 2011.

43. Chen C, Wei M, Wang C, Sun D, Liu P, Zhong X, He Q and Yu W: The histone deacetylase HDAC1 activates HIF1 $\alpha /$ VEGFA signal pathway in colorectal cancer. Gene 754: 144851, 2020.

This work is licensed under a Creative Commons Attribution-NonCommercial-NoDerivatives 4.0 International (CC BY-NC-ND 4.0) License. 